TRANS $\cdot$ núm. I3 $\cdot 2009$

DOSSIER $\cdot 19-31$
La historia del teatro traducido en la España del siglo xx está aún por escribirse. Este segmento de nuestra cultura traducida ha sido tradicionalmente ignorado en las historias del teatro español. Por suerte, lo que hace sólo veinte años se describía como un páramo investigador es hoy un terreno mucho mejor abonado y roturado. Las investigaciones sobre teatro traducido que han visto la luz progresivamente en estos años nos permitirán en breve escribir y documentar la historia del teatro traducido. Se ofrece en este artículo una visión del modo en que podría acometerse esa tarea, partiendo de lo ya investigado en el proyecto TRACE, desde la perspectiva de lo archivado por la censura (de teatro) en la época de Franco.

PalABRAS Clave: Traducción de teatro, historia de la traducción, censura, estudios descriptivos de traducción, traducción literaria inglés-español.

\title{
Traducciones (censuradas) de teatro inglés en la España de Franco. TRACE: una perspectiva histórica*
}

Raquel Merino Álvarez

Universidad del País Vasco

(UPV/EHU)
The history of translated theatre in 20th century Spain is yet to be written. As any other segment of translated culture, translated theatre is virtually absent from histories of Spanish theatre. Twenty years ago investigations on translated theatre in Spain were few and far between. Luckily the situation has changed so that we can now sketch and write a well-documented history of translated theatre. This article offers a view from the TRACE project, whose members have researched Franco's (theatre) censorship archives in the last few years.

KEYWORDS: Theatre translation, history of translation, censorship, descriptive translation studies, literary translation English-Spanish 


\section{EL PAPEL DE LOS ESTUDIOS TEATRALES DE CORTE HISTÓRICO}

20 Para mi aportación a este dossier sobre traducción teatral he optado por centrarme en uno de los ámbitos a los que he dedicado buena parte de mis esfuerzos desde que a mediados de los años ochenta Julio César Santoyo me hiciera ver que el teatro traducido no había recibido apenas atención por parte de los investigadores (Santoyo, 1994). Ya entonces opté por orientar mi investigación hacia la historia de las traducciones de teatro (inglés-español) en la segunda mitad del siglo veinte. Es decir, la perspectiva histórica era parte integral de aquel primer esfuerzo por establecer qué (obras, autores) se habían traducido, quiénes habían sido los traductores y cómo podían describirse las traducciones teatrales como hechos de la cultura meta que indudablemente eran.

$\mathrm{Ni}$ entonces contábamos ni ahora mismo contamos con una historia del teatro traducido en España. Es más, era poco menos que imposible rastrear dicho teatro en traducción en ninguna de las historias del teatro español. Por tradición, por falta de interés, o quizá por la excesiva especialización a la que se tiende en el ámbito académico, lo cierto es que no era sencillo hace veinte años tener una visión de la presencia e integración del teatro extranjero en nuestro país. Y si esto era así para el teatro publicado (tanto los textos que llegan a editarse tras un recorrido escénico, como aquéllos que fueron impresos para ser leídos) lo era aún más para el teatro representado del que siempre quedan menos vestigios, menos trazas.

* Trabajo realizado en el marco del grupo TRACE (www.ehu.es/trace). Proyecto: TRAducciones CEnsuradas INGLÉS/ALEMÁN-ESPAÑOL (TRACE I939-I985): estudios sobre catálogos y corpus. FFI2008-05479-Co2-02.
Pero, ¿̇cuáles son los márgenes de lo que se entiende por estudios sobre traducción teatral de corte histórico? A mi entender la perspectiva histórica se adopta al acometer estudios que abarcan un periodo o subperiodo, pero también cuando se rastrea la producción (en traducción) de un autor o grupo de autores, la producción de un traductor o traductores; o, por qué no, cuando se sigue la evolución de un tema específico en un periodo concretor.

Es obvio que este modo de investigar la historia de las traducciones (de teatro en España) puede llegar a parecerse a la exploración de terrenos ignotos, que necesitan ser cartografiados antes aún de poder ser sometidos a escrutinio alguno. $Y$ algo de eso puede haber cuando nos acercamos a realidades no catalogadas, de las que tenemos noticia por medio de retazos, pinceladas, que apenas pueden darnos una visión de conjunto.

\section{LA CENSURA COMO FUENTE DE INFORMACIÓN TEXTUAL Y CONTEXTUAL}

Al plantear pues un estudio diacrónico del teatro traducido se puede comenzar por inventarios o catálogos existentes, y si estos fueran escasos o incompletos, queda recurrir a otras fuentes documentales. En el caso de bibliotecas (nacional, públicas del estado o universitarias) o de bases de datos como el Index Translationum no hay otro modo que comenzar partiendo

I Por ejemplo, el tema de la homosexualidad que parece llegar al teatro español en la década de I950 a través de traducciones de teatro extranjero, preferentemente norteamericano. En Merino 2008 y 2009 se diseccionan los datos con los que contamos hasta la fecha y se esboza la cronología de la entrada de este tema en la escena española, partiendo de Un tranvía llamado deseo, pasando por Téy simpatía o Historia del zoo hasta llegar a la impactante producción de Los chicos de la banda en 1975. 
de listas predeterminadas de autores $\mathrm{u}$ obras ${ }^{2}$. Dichas listas surgirán a su vez de fuentes relacionadas con la historia literaria de los países/ lenguas objeto de estudio. De este modo se construyó un primer catálogo de traducciones de teatro representadas y publicadas (Merino, 1994).

Sin embargo cuando el investigador accede a archivos como el $\mathrm{AGA}^{3}$ para censura, las posibilidades se amplían, al tiempo que se hace más complejo el acceso a la información, por la cantidad ingente de datos y por la propia organización de los mismos. Se nos brinda en estos casos la posibilidad de ir más allá de selecciones predeterminadas de autores y obras, y se pueden acotar de forma más precisa periodos y subperiodos, generando catálogos de obras teatrales traducidas más cercanos a la realidad del teatro producido en España. Del mismo modo, es posible compilar corpus textuales más ricos y complejos: los textos teatrales publicados conviven en el AGA con manuscritos inéditos $y$ es frecuente encontrarse con diferentes traducciones de un mismo original y con diversas versiones de una misma traducción presentadas de forma sucesiva a censura.

En el contexto del proyecto TRACE, se nos presentaba por primera vez la oportunidad de acceder a un yacimiento muy poco explorado en el que el teatro producido en España y orientado a la escena (tanto original o nativo, como en traducción) había sido archivado de forma

2 En TRACE hemos accedido al Aga (Archivo General de la Administración) como principal recurso documental, pero adicionalmente hemos consultado otras fuentes como: el Index Translationum, El Libro Español, Bibliografía Española, Biblioteca Nacional (ARIADNA), Bibliotecas Universitarias (REBIUN), Bibliotecas Públicas del Estado (REBECA), ISBN; y para teatro los volúmenes de Francisco Álvaro (I958-I985) o el Centro de Documentación Teatral (CDT). Véase Bandín (2007: 94-IO2).

3 AGA, Archivo General de la Administración: <http:// www.mcu.es/archivos/MC/AGA/index.html>. exhaustiva y sistemática. De hecho ése era el objetivo del aparato censor: ejercer un control universal sobre cualquier producto que pudiera llegar al público lector o espectador. Dicho control gubernamental generó una estructura burocrática que, aparte de los fines propios para los que se creó, ha dejado un legado documental de inestimable valor. Este yacimiento documental encierra aún textos inéditos o información sobre, entre otros, el teatro que llegó a escena, o el que por diferentes motivos quedó únicamente archivado y olvidado.

El acceso a la información contenida en el AGA, para el caso del teatro, se ha realizado de dos modos: por autor o título, consultando los ficheros generados por los diferentes organismos censores 4 , o por muestreo. En el primer caso el acceso a la información es quizá más rápido, aunque la preselección de autores y obras puede condicionar el tipo de estudio que se lleva a cabo. Respecto al segundo procedimiento, el de calas o muestreo, el enorme caudal de información disponible en forma de cajas archivadoras numeradas y estructuradas según diversos criterios (llegada al archivo, tipo, origen...) nos movió a tratar de consultar, partiendo de periodos y subperiodos predeterminados, todas aquellas cajas-AGA que fuera posible, teniendo en cuenta la disponibilidad de tiempo de los investigadores involucrados y los límites establecidos por el propio archivo. De este modo se realizó un acercamiento a los fondos sobre Cultura-Teatro eligiendo de manera aleatoria aunque sistemática números de cajas-AGA identificadas como pertenecientes a un periodo (p.e. I971-I977) pero de las que ignorábamos en principio qué información (sobre obras o autores) podían contener. $\mathrm{Si}$, por poner un ejemplo, para el subperiodo I97I-I977 contábamos con la

4 Véase anexo 3. 
referencia de cajas-AGA número 85.I3I a 85.8II, elegíamos un diez por ciento, distribuidas de forma uniforme (85.I3I, 85.I4I, 85.I5I .. 85.8II), y procedíamos a solicitar, abrir y analizar el contenido de esos archivadores (carpetas organizadas por número de expediente y título, acompañadas de documentación referida al expediente y textos: libretos o ediciones).

De ese modo íbamos «cuadriculando» ciertas zonas de la superficie del mosaico correspondiente al periodo preseleccionado. Esta forma de actuar nos brindó la posibilidad de extraer del conjunto de datos, el teatro registrado por la censura, todo aquel correspondiente a autores extranjeros, y de forma exhaustiva el de autores ingleses. Además, dicho procedimiento hizo posible tener una visión global más ajustada de cuáles habían sido los autores y obras extranjeros con mayor o menor presencia y qué casos habían resultado más problemáticos o complejos desde la perspectiva de las autoridades.

Como en todo "yacimiento» mucho del material acumulado hubo de ser catalogado y analizado, y ciertos hallazgos significativos fueron inmediatamente sometidos a un escrutinio mayor: se procedió a búsquedas paralelas por autor, obra, traductor o adaptador para así poder completar la información sobre casos que ya se planteaban como representativos o prototípicos.

De este modo se confirmaba, por ejemplo, que, exceptuando clásicos como Shakespeare, Tennessee Williams había sido uno de los autores extranjeros con mayor presencia ${ }^{5}$ en el teatro

5 Conviene matizar esta afirmación: las obras de Williams, tras salvar diversas trabas censorias, llegaron al público español, tanto a través de Teatros de Cámara y Ensayo en un primer momento (años cincuenta) como mediante circuitos comerciales en las principales capitales españolas. Esta presencia se extendió desde la década de I950 hasta la de los ochenta (y hasta nuestros días) con una continuidad que da fe de su integración en el sistema español desde la década de los cincuenta (Pérez, 2004: 153). Así también surgían casos como en el de Graham Greene o Peter Shaffer, cuya producción teatral tuvo un impacto nada desdeñable en la escena española, derivado sin duda de la temática de algunas de sus obras ${ }^{6}$. De igual manera, se puede constatar que la moral sexual está en el punto de mira del aparato censor (más aún que la política), independientemente del ministro u orientación de su equipo. También se observa que, en la misma medida que se persiguen ciertos temas como la homosexualidad, diversos grupos de presión pugnan para que sean precisamente esos temas los que lleguen con más facilidad a los escenarios españoles. Y en esos casos la importación de teatro extranjero en traducción parece proporcionar mayor garantía de éxito a la hora de recabar el preceptivo permiso de representación (Merino, 2008: 243-244).

La censura española (el AGA) ha demostrado ser una fuente de gran riqueza textual y con-

teatral español. Algunos autores extranjeros consultados en el fichero AGA nos dan cifras superiores a las que encontramos para Williams en el anexo I, pero se trata de autores que quedaron restringidos a un sólo segmento del teatro español (p.e. Cámara y Ensayo), o un subperiodo específico.

6 El tema de la infidelidad en la obra de Graham Greene El amante complaciente (Merino, 2003: 662-670) provocó la repetida prohibición de la obra en un periodo de apertura. El largo proceso que comienza en 1962, culmina con la autorización con cortes que da paso al estreno en 1968. Dicho proceso ha dejado rastros documentales muy ricos: los expedientes que registran la prohibición, una cadena de textos traducidos en diversas versiones y adaptaciones, más la publicación de la obra traducida en I969.

En el caso de Peter Shaffer sus obras tocan temas espinosos: la homosexualidad en Five Finger Exercise, una obra que se cita como antecedente de introducción del tema en el teatro español (Álvaro, 1975: 86); o la leyenda negra (sobre la conquista del Perú) en The Royal Hunt of the Sun, que choca con una actitud involucionista en el periodo I969-1975. Y es otra obra de Shaffer, Equus, la que da lugar al primer desnudo masculino y femenino (autorizado) en los escenarios españoles (ib: : IO7-III). 
textual para poder estudiar el segmento de la cultura española que nos ocupa: la traducida. $\mathrm{El}$ hecho de que en el proceso de archivo no se diferenciara producción nativa ${ }^{7}$ de producción en traducción nos permite observar el sistema teatral de una forma integral. Del mismo modo, podemos acceder a aquella cultura potencial que no llegó a representarse (o publicarse) pero que en un momento dado pudo haber llegado a existir. Contamos con suficiente información contextual y textual para reconstruir también esa cultura inédita, esas páginas olvidadas.

Por último quisiera apuntar otra de las ventajas que conlleva investigar desde la perspectiva de los archivos generados por la censura franquista. Cuando hablamos de los textos que se presentaron a censura para conseguir el correspondiente permiso de representación en los años cuarenta, cincuenta, sesenta o setenta pensamos quizá erróneamente que se trata de textos ya superados y obsoletos. Y lo mismo podríamos pensar de los libros publicados. Nada más lejos de la realidad. De la ingente producción en traducción (de teatro, narrativa o cine) que se generó en el periodo franquista, sólo una mínima parte se ha retraducido. La mayoría sigue utilizándose (para su publicación y representación), en la versión que quedó fijada en formato impreso en su época, con mínimos cambios ${ }^{8}$. Por tanto estamos ante una historia

7 La diferenciación entre texto «nativo» $\mathrm{y}$ «traducido» se hace necesaria en investigaciones como las aquí esbozadas. «Texto nativo (producido en la lengua o cultura meta que se estudia): por definición los textos nativos se perciben como 'no-traducción'. Los textos nativos conviven con las traducciones de otras lenguas en un mismo contexto meta» (Rabadán y Merino, 2004: 25). En la producción de dramaturgos españoles como Pemán o Gala se encuentran ambos tipos, mientras que la de Vicente Balart o Catalina Montes consta únicamente de traducciones. Véase anexo 2.

8 De los muchos ejemplos que podríamos citar el de la novela popular del oeste, publicada en grandes tiradas en los cincuenta y sesenta, y reeditada en los últimos años, muy presente en nuestra cultura que nos lleva a plantearnos la presencia en nuestros días de aquellos productos teatrales, cinematográficos, o libros impresos traducidos y censurados, reutilizados y revisados.

Hasta aquí he esbozado de manera muy sucinta el modo en que se ha intentado abordar la cuestión del teatro traducido en España desde la perspectiva histórica que brindan los archivos sobre censura. También he querido subrayar la importancia de recurrir a fuentes documentales tradicionales al tiempo que se explora el gran potencial que encierran otros fondos como el AGA.

Quizá sea ahora el momento de hacer un breve repaso por lo compilado hasta la fecha en el entorno TRACE-teatro. Los antecedentes de este modo de catalogar traducciones de teatro, como paso previo a la selección de corpus textuales objeto de estudio, los encontramos en Merino (I994). Más adelante se planteó como objetivo catalogar el teatro inglés traducido en el primer tercio del siglo $\mathrm{xx}^{9}$, una catalogación que llevó a su autora a enfocar la investigación de su tesis doctoral al periodo I939-I963 (Pérez, 2004), dentro del proyecto TRACE. El teatro traducido del siguiente periodo, hasta $1985^{\text {Io }}$,

supuestamente sin modificación alguna, es muy representativo. En el caso específico del teatro, son pocas las obras que hasta la fecha hemos podido constatar que hayan sido totalmente retraducidas, más bien abundan las reediciones, las revisiones e incluso los plagios de traducciones anteriores. Quizá un ejemplo menos extremo, pero muy significativo, pueda servir de botón de muestra a este respecto: la traducción 'literal' de Historia del zoo de Edward Albee, presentada a censura en 1962 , difiere muy poco del texto revisado por su traductor, William Layton, para la primera edición de la obra en I99I (Merino, 2003: 658-659).

9 María Pérez López de Heredia, Las traducciones españolas del teatro inglés (1898-r939). <http://www.ehu.es/ trace/tesisytrabajos-tracedea.html $>$.

Iо En las investigaciones TRACE-teatro se incluye el decenio I975-I985 no sólo porque las traducciones que se aprueban, representan y publican en años anteriores siguen 
Catálogos y corpus

Pre-trace

TRACEti

TRACEti

TRACEti

TRACE-teatro

I950-I990

I939-I963

I96I-I985

I939-I985

No registros/

I56

225

650

678

traducciones

catálogo

(559 public.

II9 repres.)

\begin{tabular}{|c|c|c|c|c|}
\hline $\begin{array}{l}\text { Corpus pre-textual } \\
\text { TO-TM }\end{array}$ & $\begin{array}{l}\text { Ioo } \\
\text { bi-textos } \\
(\mathrm{TO}+\mathrm{TM})\end{array}$ & $\begin{array}{l}\text { I7 } \\
\text { Conjuntos } \\
(\mathrm{TO}+\mathrm{TMn})\end{array}$ & $\begin{array}{l}50 \\
\text { Conjuntos } \\
(\mathrm{TO}+\mathrm{TMn})\end{array}$ & \\
\hline $\begin{array}{l}\text { Corpus textual }^{\mathrm{a}} \\
\text { TO-TM }\end{array}$ & $\begin{array}{l}{ }_{4} \text { Conjuntos } \\
\text { textuales } \\
(5 \mathrm{TO}+7 \mathrm{TM})^{\mathrm{b}}\end{array}$ & $\begin{array}{l}{ }_{4} \text { Conjuntos } \\
\text { textuales } \\
(4 \mathrm{TO}+\mathrm{I} 5 \mathrm{TM})^{\mathrm{c}}\end{array}$ & $\begin{array}{l}3 \text { Conjuntos } \\
\text { textuales } \\
(3 \mathrm{TO}+\mathrm{I} 2 \mathrm{TM})^{\mathrm{d}}\end{array}$ & $\begin{array}{l}6 \text { Conjuntos } \\
\text { textuales } \\
(6 \mathrm{TO}+30 \mathrm{TM})^{\mathrm{e}}\end{array}$ \\
\hline
\end{tabular}

a Una vez analizada la información recogida en los catálogos TRACE, se seleccionan, mediante criterios debidamente justificados y derivados de dicho análisis, una serie de corpus textuales que podrán ser pre-textuales (tenemos acceso a fragmentos del texto o referencias al propio texto pero no hemos podido reconstruir la cadena textual completa) o textuales (disponemos de una cadena textual completa del tipo то>TмI, тм2..., y por tanto podemos efectuar la comparación de bi-textos то-тм y/o de parejas тм-тм).

b Panorama desde el puente de Arthur Miller (2TO + 2TM), Vengan corriendo que les tengo un muerto de Jack Popplewell (то + тм), Mulato de Langston Hughes (Alfonso Sastre) (то + 2тм), Pasión de Edward Bond (Carla Matteini) (то + тм). (Merino, 1994: 79-180).

c TRACEti 1939-1963. Un tranvía llamado Deseo / A Streetcar Named Desire (то + 6тм), La gata sobre el tejado de zinc / A Cat on a Hot Tin Roof (то + 4Tм) у Dulce pájaro de juventud/Sweet Bird of Youth (то + 2тм) de Tennessee Williams; y Deseo bajo los olmos / Desire Under the Elms, de Eugene O'Neill (то + 3тм). (Pérez López de Heredia, 2004: 209-45I).

d TRACEti 196I-1985. El amante complaciente de Graham Greene (то + 5TM), Historia del zoo de Edward Albee (To + 3TM), Los chicos de la banda de Mart Crowley (то + 4TM). (Merino 2003, 2005a, 2005b, 2008).

e TRACEti 1939-1985. La transición del catálogo al corpus en Bandín (2007) da como resultado una selección de seis conjuntos textuales, de los cuales tres corresponden a obras de Shakespeare «aquellas traducciones de sus obras que tuvieron mayor repercusión en el sistema teatral... con mayor número de traducciones o versiones distintas: Hamlet (Io), La Fierecilla Domada (Io) y Otelo (II)» (Bandín, I6I-I77). Completan el corpus 5TM de Volpone, las traducciones de Los lunáticos de Middleton y Rowley y de 'Tis a Pity She's a whore de John Ford.

lo aborda Merino. La última aportación en el seno del grupo ha sido la tesis doctoral de Elena Bandín (2007) sobre teatro clásico inglés en traducción. En ella la autora analiza el periodo 1939-I985 en un estudio que explora de forma

estando presentes en el sistema teatral español en la década de los ochenta; sino sobre todo porque la estructura burocrática que sustentaba el aparato censor sigue funcionando de un modo similar, bajo diferentes etiquetas (censura > ordenación > calificación), registrando información sobre solicitudes para representar obras teatrales. Este modo de funcionar culmina en mayo de 1985 con la reorganización del Ministerio de Cultura de modo que, al desaparecer las secciones correspondientes, dejan de generarse archivos y expedientes (Merino, 2000). exhaustiva el teatro clásico inglés. En el cuadro superior ofrecemos algunos de los datos de cada catálogo y corpus ya estudiado.

En los últimos años se han publicado otros estudios sobre teatro a partir de investigaciones en el AGA, centrados en dramaturgos españoles. Así Muñoz Cáliz (2005) cataloga la producción de los escritores teatrales españoles críticos con el régimen y Catherine O'Leary (2005) analiza en profundidad la obra de Buero Vallejo. Este tipo de estudios podrán servir en el futuro para contrastar información sobre la producción en traducción de los autores españoles de los que hubiera datos. En todo caso la utilidad de 
estudios realizados desde la perspectiva de la investigación sobre teatro español se ve limitada por dos circunstancias. En primer lugar, siendo el centro de interés la producción «original» de los dramaturgos seleccionados, las traducciones que dichos autores pudieran haber suscrito no se registran o analizan sistemáticamente. Otro factor a tener en cuenta es la propia selección de un dramaturgo o un grupo como objeto de estudio, incluso en estudios ambiciosos, centrados en un amplio abanico de autores, como el de Muñoz Cáliz, que no son ni pretenden ser exhaustivos o representativos de todas las tendencias. Lo mismo podría decirse de obras como las de O'Leary (2005) que parten de una tradición académica que resalta una imagen del teatro español como un teatro en «versión original», y por tanto soslaya el segmento de la cultura teatral española que coexistió con aquél: el teatro traducido.

Y puesto que parece que sólo desde la perspectiva de los Estudios de Traducción interesa dar cuenta de esa otra historia (invisible) del teatro español, la investigación sobre teatro traducido partiendo de una preselección de nombres de autores habrá de hacerse de forma directa en los archivos si se quiere llegar a dar una visión completa de este tipo de producción. Las búsquedas guiadas tendrán que centrarse en aquellos dramaturgos españoles con mayor número de traducciones realizadas, un extremo que vendría corroborado por calas sistemáticas en inventarios exhaustivos como el del AGA, que permitirían abordar el colectivo de traductores o adaptadores con poca o ninguna producción original ${ }^{\mathrm{II}}$.

II En el anexo 2 incluimos muestras de búsquedas efectuadas partiendo de nombres de dramaturgos españoles que firmaron traducciones (p.e. Pemán) o traductores con escasa (o nula) producción original (p.e. Balart).

\section{HACIA UNA HISTORIA DEL TEATRO TRADUCIDO EN ESPAÑA}

Si reunimos los datos con los que contamos en la actualidad parece que ya podríamos pensar en acometer el esbozo de una Historia del Teatro Traducido en España en el siglo xx. Esta historia comenzaría, a mi entender, refundiendo los catálogos ya compilados en una base de datos global donde se registre el teatro representado y el publicado. Dicha base de datos se habría alimentado de forma directa o indirecta de archivos como el AGA, bibliotecas, compendios y estudios anteriores. También convendría acudir a estudios menos académicos pero tremendamente informativos si se contrasta la información que aportan ${ }^{\mathrm{I} 2}$. No hay duda de que se podría reconstruir la historia teatral y/o editorial de autores u obras concretos, pero también de teatros, directores, traductores, adaptadores o editoriales. En algunos casos, donde la investigación que sustenta los datos haya sido más exhaustiva y se hayan recopilado los textos, podríamos incluso tener acceso a los correspondientes corpus textuales.

En todo caso, con algún esfuerzo y cierta coordinación, ahora sí que sería posible llegar a dar una visión bastante cercana del devenir del teatro traducido en España con datos contrastados. Está claro que habrá autores de los que contaremos con mucha más información: aquellos a los que se han dedicado más esfuerzos y en los que se han centrado trabajos más específicos $^{13}$. No es menos cierto que de aquellos

I2 Memorias como la de Adolfo Marsillach (Tan lejos, tan cerca. Mi vida, Barcelona: Tusquets, I998) o José María García Escudero (La primera apertura. Diario de un director general, Barcelona: Planeta, 1978).

I3 El panorama de los estudios sobre traducción dramática en España cuenta con referentes claros. Para estudios sobre Shakespeare es ineludible mencionar la labor continuada del Instituto Shakespeare, con Manuel Á. Conejero al frente, o las investigaciones centradas en la 
autores que tengamos referencias mínimas al menos éstas servirán para certificar que existieron en traducción al español ${ }^{\mathrm{I} 4}$. Muchos autores considerados comerciales, o simplemente otros con una corta vida en los escenarios de nuestro país, podrían no haber llegado a publicarse, y por tanto habrían dejado pocos rastros en nuestra cultura. De ellos, posiblemente gracias a investigaciones centradas en los archivos de censura, tendríamos datos suficientes para poder facilitar futuras investigaciones, incluso de carácter textual.

Pero, antes de proceder a refundir catálogos existentes, aún está pendiente la catalogación de las traducciones firmadas por dramaturgos españoles de una forma sistemática y con afán de exhaustividad. A dicho catálogo, habría que añadir el de traductores y adaptadores para el que tenemos ya una buena aproximación (los catálogos de traducciones basados en listados de autores extranjeros) pero cuya imagen sería mucho más nítida al proceder a efectuar búsquedas completas por nombre de traductor-adaptador o incluso director. Los anexos finales tratan de apuntar cuál sería la naturaleza de la información derivada de búsquedas como las esbozadas.

Universidad de Murcia (Pujante y Campillo, 2007; Campi1lo, 2005; SHESTRA <http://cvi.cpd.ua.es/shestra/>). Dicha labor unida a aportaciones como la de Bandín (2007) o Zaro (2007), dan fe del trabajo realizado sobre la obra en traducción del dramaturgo inglés. Por su parte Rafael Portillo (Universidad de Sevilla) ha aglutinado investigaciones sobre teatro en traducción de indudable interés (Fernández Quesada, 2007). Del mismo modo Marta Mateo en la Universidad de Oviedo ha dirigido investigaciones como las de Braga Riera (2006) o Serrano (2003) que han contribuido a enriquecer un panorama variopinto que Pilar Ezpeleta (2007: I39-160) detalla en su volumen Teatro y Traducción.

I4 Alan Ayckbourne, o Robert Bolt, cuentan con apenas dos obras registradas en el fichero AGA. Otros autores más comerciales, como Terence Rattigan, que sí fueron traducidos, representados y publicados en muchas más ocasiones, no han sido objeto de estudio, aunque habría que plantearse si deberían serlo.

Se trata por tanto de colaborar, en la medida de lo posible de forma coordinada, para conseguir hacer que la imagen del mosaico (el teatro español en traducción) que ya se ha ido cuadriculando y analizando, pueda ser cada vez más nítida, y refleje de forma más fidedigna esa parte integral del teatro español, el traducido, que, si no ignorado, al menos ha sido relegado en el ámbito académico ${ }^{\mathrm{I}}$.

ANEXO I. Autores extranjeros en inventario $A G A$ (03) 046.000

\begin{tabular}{lcc} 
autor & n. obras & fechas \\
\hline Shakespeare, W. & 74 & I940-I978 \\
Williams, T. & 26 & I945-I974 \\
Pinter, Harold & 23 & I96I-I98I \\
Priestley, J. B. & 22 & I945-I984 \\
Rattigan, T. & I6 & I955-I98I \\
Maugham, Somerset & I5 & I940-I966 \\
Rattigan, T. & I4 & I955-I972 \\
Christie, Agatha & I4 & I949-I97I \\
Albee, E. & I3 & I963-I976 \\
Miller, A. & I2 & I95I-I974 \\
Hamilton, Patrick & II (2 TO) & I944-I982 \\
Shaffer, Peter & II & I959-I983 \\
Greene, Graham & 9 & I953-I978 \\
Dickens, Charles & 4 & I95I-I983 \\
Shaffer, Anthony & 3 & I970-I983 \\
Bolt, Robert & 2 (I TO) & I962 \\
\hline
\end{tabular}

Selección de resultados: búsqueda guiada - autor, número de obras registradas y fechas - en inventario AGA (03) 046.000

I5 Quisiera enlazar lo dicho aquí con una afirmación (Merino, 2008: 246) que cito como arranque a «La historia de las traducciones de teatro inglés en España en el siglo xx: perspectiva desde el proyecto TRACE», mi contribución al volumen Lengua, traducción, recepción. En honor de Julio César Santoyo: «por todo lo dicho urge clamar por la necesidad de una historia del teatro español integral, que acompase producción original y traducida sin expurgo ni selección previa, integrando el canon completo de dramaturgos-traductores, traductores y adaptadores, esto es, teniendo en cuenta la vida escénica española tal y como aconteció». 


\section{título}

Un tranvía llamado deseo

El zoo de cristal

Verano y Humo

El ángel de piedra

Figuretes de vidre

Un tranvía llamado Deseo

La rosa tatuada

Una gata sobre un tejado de zinc...

Camino real

Una gata sobre un tejado de zinc...

La caída de Orfeo

Dulce pájaro de juventud

Hasta llegar a entenderse

La noche de la iguana

El caso de las petunias pisoteadas

El largo adiós

El más extraño idilio

La marquesa de Larkspurtlotion

Auto da fe

Háblame de la lluvia y déjame escuchar

Lo que no se dice

Cena desagradable

Repentinamente el pasado verano

Veintisiete vagones de algodón

La marquesa de Larkspurtlotion

Súbitamente el último verano exped./año fecha inic. fechafin. otros

\begin{tabular}{|c|c|c|l|c}
\hline 0217/50 & I950 & I957 & $\begin{array}{l}\text { Méndez Herrera, José/ } \\
\text { Fresno, Maruchi - Guerrero. }\end{array}$ & Sí
\end{tabular}

\begin{tabular}{|l|l|l|}
\hline $0274 / 50$ & I950 & 1978 \\
\end{tabular}

Vázquez Vigo, Carme/

Gordon, J.-De Quinto, $\mathrm{M}^{\mathrm{a}}$

\begin{tabular}{|r|r|r|l|r}
\hline 0342/52 & I952 & I96I & Montes, Conchita & S \\
\hline 0199/55 & I955 & I955 & Cabo, Antonio de (adap.) & S \\
\hline O134/56 & I956 & I966 & & S \\
\hline
\end{tabular}

\begin{tabular}{l|l|l|l|l}
\hline $0300 / 56$ & 1956 & 1956 & Guerrero Zamora, Juan & S
\end{tabular}

\begin{tabular}{|l|l|l|l|l}
\hline $0015 / 57$ & I957 & I958 & Cabo, Antonio de (adap.) & Sí
\end{tabular}

\begin{tabular}{|l|l|l|l|l|}
\hline $0228 / 58$ & I958 & I958 & Frade Almohalia, José \\
\hline
\end{tabular}

\begin{tabular}{|l|c|c|l|c}
\hline $0003 / 58$ & $195^{8}$ & $195^{8}$ & Vila Selma, Enrique & $\mathrm{S}$ \\
\hline
\end{tabular}

\begin{tabular}{|l|l|l|l|l|}
\hline oor7/59 & I959 & I962 & Orce, Ramón & Sí \\
\hline
\end{tabular}

\begin{tabular}{|l|l|l|l|}
\hline 0о03/60 & I960 & 1968 & Sí \\
\hline
\end{tabular}

\begin{tabular}{|l|l|l|l|r|}
\hline or52/60 & I960 & I962 & Alonso, Justo & Sí \\
\hline or67/63 & I963 & I965 & Lorente Muñoz, Rafael & \\
\hline
\end{tabular}

\begin{tabular}{|l|r|r|r|r|}
\hline $0007 / 64$ & I964 & I967 & \\
\hline oI77/68 & I968 & I968 & \\
\hline
\end{tabular}

(2)

\begin{tabular}{|c|c|c|l|c}
\hline $0356 / 68$ & I968 & I972 & & Sí \\
\hline $0395 / 68$ & I968 & I969 & & Sí \\
\hline $0176 / 68$ & I968 & I970 & López de Cervera, M. Dolores & Sí \\
\hline $0369 / 69$ & I969 & I969 & Adán Sánchez, Pedro & Sí \\
\hline $0027 / 69$ & I969 & I972 & Espada Díaz, José de & \\
\hline $0497 / 70$ & I970 & I970 & López Cervera, Dolores & \\
\hline $0651 / 71$ & I971 & I971 & & Sí \\
\hline $0256 / 71$ & I971 & I971 & Borrel, Carlos & Sí \\
\hline $0339 / 72$ & I972 & I972 & & Sí \\
\hline $0516 / 72$ & I972 & I972 & San Miguel Sánchez, F. ${ }^{\text {co }}$ & Sí \\
\hline $0554 / 74$ & I974 & I974 & & \\
\hline
\end{tabular}

«Tennessee Williams» en inventario AGA (03)046.0oo 
título

\begin{tabular}{|c|c|c|c|c|c|c|}
\hline titulo & $\exp / a \tilde{n} o$ & dictamen & teatro & $\begin{array}{l}\text { traductor } \\
\text { adaptador }\end{array}$ & editorial & $\begin{array}{l}\text { año } \\
\text { public. }\end{array}$ \\
\hline El zoológico de cristal & $/ 45$ & & & Mirlas, León & & I953 \\
\hline Verano y humo & $/ 48$ & & & $\begin{array}{l}\text { Mirlás, León y Bar- } \\
\text { berá, Manuel }\end{array}$ & Losada & I979 \\
\hline \multirow[t]{4}{*}{ Un tranvía llamado deseo } & $217 / 50$ & Autorizada & Reina Victoria & Méndez Herrera, José & Escelicer & I962 \\
\hline & $217 / 50$ & $\begin{array}{l}\text { Teatro de Cáma- } \\
\text { ra Oficial }\end{array}$ & $\begin{array}{l}\text { Cámara del } \\
\text { Español }\end{array}$ & Méndez Herrera, José & Alfil & I962 \\
\hline & $217 / 50$ & $\begin{array}{l}\text { Autorizada Tea- } \\
\text { tro de Cámara }\end{array}$ & & Méndez Herrera, José & Alfil & I962 \\
\hline & $217 / 50$ & Prohibida & Barcelona & Méndez Herrera, José & Alfil & I962 \\
\hline $\begin{array}{l}\text { Kristalesko iruditxoak } \\
\text { (vasco) }\end{array}$ & $485 / 62$ & $\begin{array}{l}\text { Autorizada } \\
\text { (sesión única) }\end{array}$ & $\begin{array}{l}\text { Principal (S. } \\
\text { Sebastián) }\end{array}$ & Beobide, Ignacio & & \\
\hline $\begin{array}{l}\text { La gata sobre el tejado } \\
\text { de zinc caliente }\end{array}$ & $554 / 79$ & $\begin{array}{c}\text { «Calificación» } \\
+\mathrm{I} 4\end{array}$ & Marquina & Diosdado, Ana & $\begin{array}{c}\text { MK } \\
\text { Ediciones }\end{array}$ & I984 \\
\hline \multirow[t]{2}{*}{ El zoológico de cristal } & $\mathrm{I} 5 \mathrm{O} / 8 \mathrm{I}$ & & Marquina & Vázquez Vigo, C. & & \\
\hline & I5o/8I & Calificac. + I4 & & Schurjin & & \\
\hline $\begin{array}{l}\text { No puedo imaginar } \\
\text { mañana }\end{array}$ & $\mathrm{II} 3 / 83$ & Calificac. + I4 & & $\begin{array}{l}\text { Tabares Soriano, } \\
\text { Mercedes }\end{array}$ & & \\
\hline $\begin{array}{l}\text { Advertencia per a } \\
\text { embarcacion petites }\end{array}$ & $59 / 83$ & Calificac. + I6 & & Melendres, Jaume & & \\
\hline El zoológico de cristal & $403 / 83$ & $\begin{array}{l}\text { Calificac. sin res- } \\
\text { tric. de edad }\end{array}$ & & Gordon, José & Escelicer & 1964 \\
\hline $\begin{array}{l}\text { Veintisiete vagones de } \\
\text { algodón }\end{array}$ & $\mathrm{I} 46 / 83$ & Calificac. + I6 & & $\begin{array}{l}\text { López Cervera, Ma } \\
\text { Dolores }\end{array}$ & & \\
\hline $\begin{array}{l}\text { La gata sobre el tejado } \\
\text { de zinc caliente }\end{array}$ & $37 / 84$ & Autorizada +I4 & $\begin{array}{l}\text { Reina Victo- } \\
\text { ria de Madrid }\end{array}$ & $\begin{array}{l}\text { Gandolfo, Carlos y } \\
\text { Maldonado, Salv. }\end{array}$ & & \\
\hline El zoológico de cristal & II $8 / 84$ & $\begin{array}{l}\text { Autorizada sin } \\
\text { restric. de edad }\end{array}$ & $\begin{array}{l}\text { En Sástago } \\
\text { (Zaragoza) }\end{array}$ & Mirlas, León & & \\
\hline $\begin{array}{l}\text { En el bar de un hotel } \\
\text { de tokio }\end{array}$ & $284 / 84$ & Autorizada $+\mathrm{I} 6$ & $\begin{array}{l}\text { Madrid y gira } \\
\text { por España }\end{array}$ & $\begin{array}{l}\text { Producciones Divi- } \\
\text { nas }\end{array}$ & & \\
\hline Un tranvía llamado deseo & & & & Llovet, Enrique & MK Edic. & I988 \\
\hline
\end{tabular}

I6 <http://www.ehu.es/trace/catalogos.html>. Para esta tabla se han seleccionado 8 de los 35 campos que conforman la ficha del catálogo TRACEti (Merino, 200I; y Pérez L. de Heredia, 2005). De las 59 entradas para Tennessee Williams registradas en TRACEti sólo se reproducen aquí los registros que no aparecen al realizar la consulta en la base de datos AGA (03) 046 .00o. Hemos podido observar discrepancias en los ficheros manuales AGA (título y autor) que manejaba la propia sección de censura de teatro. Posteriormente en la fase de búsquedas por muestreo de forma directa en las cajas archivadoras recogimos datos sobre autores u obras que no aparecían en dichos ficheros. Esto, unido a la información adicional sobre ediciones de obras teatrales, da lugar a la información que se recoge en esta tabla sobre Tennessee Williams. 


\begin{tabular}{|c|c|c|c|}
\hline autor & \begin{tabular}{|c|}
$n^{\circ}$ obras en \\
AGA ori- \\
gionales
\end{tabular} & $\begin{array}{c}n^{\circ} \text { obras } \\
\text { en } A G A \\
\text { traducc. }\end{array}$ & fechas \\
\hline Pemán, J. M. & 64 & 7 & I939-I976 \\
\hline López Rubio, J. & 24 & I8 & I949-I972 \\
\hline Salom, Jaime & 23 & 3 & I948-I976 \\
\hline Buero, A. & 23 & ० & I949-I978 \\
\hline Marsillach, A. & $\mathrm{I} 2$ & I5 & I955-1976 \\
\hline Escobar, Luis & II & I8 & I944-I97I \\
\hline Alonso, J. L. & 3 & 42 & I947-I973 \\
\hline Balart, Vicente & I & I8 & I95I-I972 \\
\hline Arteche, J. J. & ○ & I7 & I96I-I977 \\
\hline Montes, Conchita & ० & I6 & $1945^{-1978}$ \\
\hline Matteini, C. & o & 4 & I963-I977 \\
\hline
\end{tabular}

Selección de resultados: buisqueda guiada «autores y/o traductores»: dramaturgos (Pemán, López Rubio, Salom, Buero), directores (Marsillach, Escobar, Alonso), traductores y adaptadores (Matteini, Balart, Arteche) y actores (Montes), en inventario $A G A$ (03) 046.000

\section{ANEXO 3}

\section{modelo de ficha "autor" $A G A$ :}

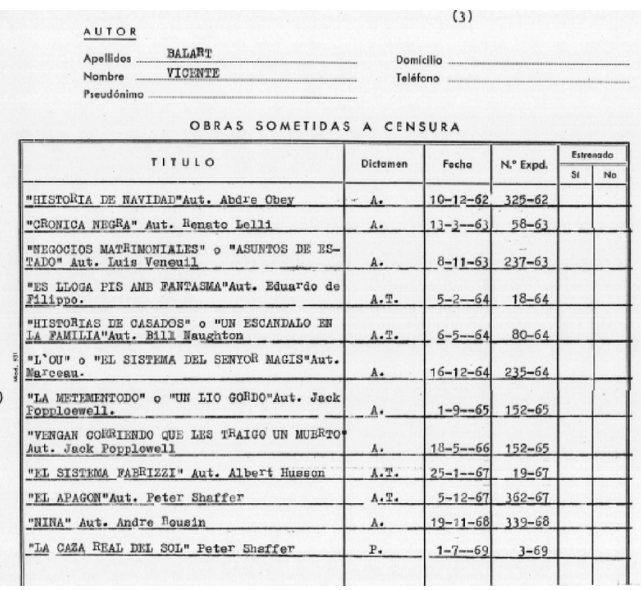

\begin{tabular}{|c|c|c|c|c|c|}
\hline \multirow{2}{*}{ TITULO } & \multirow{2}{*}{ Distamen } & \multirow{2}{*}{ Focho } & \multirow{2}{*}{ N. Expd. } & \multicolumn{2}{|c|}{ Estrendo do } \\
\hline & & & & 51 & No \\
\hline "HISTORIA IIE MAVIDAD"Aut. Abdre Obey & $-\Lambda$. & $10-12=62$ & $325-62$ & & \\
\hline "ORRONICA NEGRA" Aut. Fenato Lolli & A. & $13-2-63$ & $58-63$ & & \\
\hline 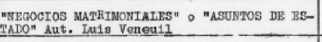 & A. & $-8=11-63$ & $237-63$ & & \\
\hline 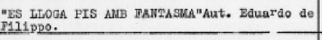 & A.T. & $5-2-64$ & $18-64$ & & \\
\hline 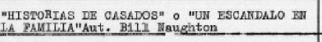 & A.T. & $6-5=$ & $80-64$ & & \\
\hline $\begin{array}{l}\text { "L'OU" o "BL SISTEKA DEL SERYOR MAGIS"Aut. } \\
\text { Ygreeau. }\end{array}$ & A. & $16-12-64$ & $235-64$ & & \\
\hline 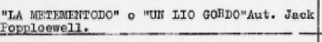 & A. & $1-9--65$ & $152-63$ & & \\
\hline 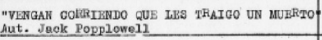 & A. & $18-5=-66$ & $152-65$ & & \\
\hline "IL SISTiana BABRIZzI * Aut. Albert Husson & A.T. & $25-1--67$ & $19=67$ & & \\
\hline EL APAGCOE"Aut. Reter Shoreer & - A.I. & $5-12-67$ & $362-67$ & & \\
\hline "nimA" Aut. Andre Rouoin & A. & $19-11-68$ & $339-68$ & & \\
\hline "IA CAZA RBAL DHL SOL" Peter Shaffer & P. & $1-7-69$ & $3-69$ & & \\
\hline
\end{tabular}

\section{modelo de ficha «titulos» $A G A$}

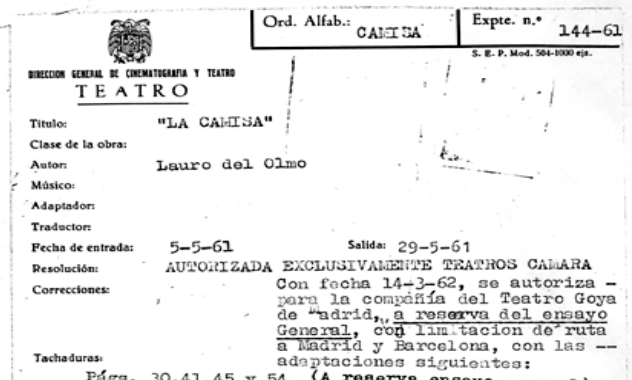

Libro Cágs: $30,41,45$ y 54 . (A reaerva ensayo general) - Ho raciablo.

\section{DATOS DE LA OBRA}

Compañat DrDO, Poquefio Teatro Teatro de estreno:

Pechas

Clasticación de la obra: AUNOIIZADA thatoron to 28 afros.

Titulos anteriores:

Censor Dolfo Carril. Rvdo, Tadre Avelino

\section{OBSERVACIONES}

Por oxden del Diroctor General co:znicada personal y ve balnente al Jefe de la Sección de 'leatro, esta puede au rizarse para 'seatros de Cánara, sin tachaduras.

Concofolya 14 de marzo de 1.962 , 59 a utoziza para repres Diroctor Genoral do c. J T. Bin limitación de ruta
RECIBIDO DICEMBRE 2008 ACEPTADO FEBRERO 2009 


\section{REFERENCIAS BIBLIOGRÁFICAS}

Álvaro, F. (1975) [1958]. El Espectador y la crítica. El teatro en España en 1975, Valladolid: Edición del autor. [Volúmenes anuales I958-1985.]

Bandín Fuertes, E. (2007). Traducción, recepción y censura de Teatro clásico inglés en la España de Franco. Estudio descriptivo-comparativo del Corpus TRACEtci (1939-I985). Tesis doctoral, León: Universidad de León.

Braga Riera, J. (2006). La traducción al inglés en el siglo XVII: las comedias del siglo de oro español, Tesis doctoral, Oviedo: Universidad de Oviedo.

Campillo Arnaiz, L. (2005). Estudio de los elementos culturales en las obras de Shakespeare y sus traducciones al español por Macpherson, Astrana y Valverde, Tesis doctoral, Murcia: Universidad de Murcia.

Ezpeleta Piorno, P. (2007). Teatro y traducción. Aproximación interdisciplinaria desde la obra de Shakespeare, Madrid: Cátedra.

Fernández Quesada, M. N. (2007). Evolución de la obra teatral de Samuel Beckett en los escenarios españoles: 1955-2000. Censura, representación y crítica, Tesis doctoral, Sevilla: Universidad Pablo Olavide.

Gutiérrez Lanza, C. (2007). «La labor del equipo TRACE: metodología descriptiva de la censura en traducción», en R. Merino, J. M. Santamaría, E. Pajares (eds.) Trasvases culturales: literatura, cine y traducción, 4, Bilbao: Universidad del País Vasco, pp. 55-64. <http://www.represura.es/ represura $>$ [Consulta: 4 octubre 2008].

Mateo Martínez Bartolomé, M. (1995). La traducción del humor: las comedias inglesas en español, Oviedo: Universidad de Oviedo.

Merino Álvarez, R. (1994). Traducción, tradición y manipulación. Teatro inglés en España 1950-90, León: Universidad de León y Universidad del País Vasco.

Merino Álvarez, R. (2000). «El teatro inglés traducido desde I960: censura, ordenación, calificación», en R. Rabadán (ed.) Traducción y censura inglésespañol, I939-I985. Estudio Preliminar, León: Universidad de León, pp. I2I-I5I.

Merino Álvarez, R. (200I). «Presentación de la base de datos TRACE (Traducciones Censuradas inglés-español)», en E. Pajares, R. Merino y J. M. Santamaría (eds.) Trasvases Culturales. Literatura, cine y traducción, 3, Bilbao: Universidad del País Vasco, UPV/EHU, pp. 287-295.

Merino Álvarez, R. (2003). «TRAducciones CEnsuradas inglés-español: del catálogo al corpus TRACE (teatro)», en R. Muñoz (ed.) Primer congreso internacional de la Asociación Ibérica de Estudios de Traducción e Interpretación, AIETI, Granada: Universidad de Granada, pp. 64I-670. [CD-rom, ISBN 84-93360-o-9].

Merino Álvarez, R. (2005a). «From catalogue to corpus in DTS. Translations censored under Franco: the TRACE Project». Revista Canaria de Estudios Ingleses, 5I, pp. 84-I04.

Merino Álvarez, R. (2005b). «La investigación sobre teatro inglés traducido inglés-español, I9942004". Cadernos de Literatura Comparada, I2/13, diciembre, pp. 99-I20. <http://www.ehu.es/trace/ publicaciones/2005cRMA_Cadernos.pdf $>$ [Consulta: 4 febrero 2009].

Merino Álvarez, R. (2008). «La homosexualidad censurada: estudio sobre corpus de teatro TRACEti (desde 1960)", en R. Merino (ed.) Traducción y censura en España (1939-I985). Estudios sobre corpus TRACE: cine, narrativa, teatro, Bilbao: Universidad del País Vasco y Universidad de León, pp. 243286. <http://www.ehu.es/servicios/se_az/trace. pdf $>$ [Consulta: 4 febrero 2009].

Merino Álvarez, R. (2009). «Building TRACE (translations censored) theatre corpus: some methodological questions on text selection», en M. Muñoz (ed.) Translation and Cultural Identity: Selected Essays on Translation and Cross-Cultural Communication, Newcastle: Cambridge Scholars Publishing.

Muñoz Cáliz, B. (2005). El teatro crítico español durante el franquismo visto por sus censores, Madrid: Fundación Universitaria Española.

O'Leary, C. (2005). The theatre of Antonio Buero Vallejo: ideology, politics and censorship, Woodbridge: Támesis.

Pérez López de Heredia, M. (2004). Traducciones censuradas de teatro norteamericano en la España de Franco (1939-I963), Bilbao: Universidad del País Vasco.

Pérez López de Heredia, M. (2005). «Inventario de las traducciones censuradas de teatro norteamericano en la España de Franco (I939-I963)», en R. Merino, J. M. Santamaría, E. Pajares (eds.) 
Trasvases culturales: literatura, cine y traducción, 4, Bilbao: Universidad del País Vasco, pp. 97-II2.

Pujante, Á. L. y L. Campillo (eds.), (2007). Shakespeare en España. Textos I764-i916. Introducción y notas de Á. L. Pujante, Granada y Murcia: Universidad de Granada y Universidad de Murcia.

Rabadán, R. y R. Merino (2004). «Introducción», en

G. Toury. Los estudios descriptivos de traducción, y más allá. Metodología de la investigación en estudios de traducción. (Descriptive Translation Studies, and beyond). Traducción, introducción y notas de $\mathrm{R}$. Rabadán y R. Merino, Madrid: Cátedra, pp. I7-33. Santoyo, J. C. (1994) «Introducción», en R. Merino. Traducción, tradición y manipulación. Teatro inglés en España, I950-I990, León: Universidad de León/Lejona y Universidad del País Vasco, pp. 9-I4.

Serrano, M. J. (2003). «La traducción al español de las referencias culturales en Who's Afraid of Virginia Woolf? de Edward Albee». Translation Journal, 7/4. <http://accurapid.com/journal/26literi. htm> [Consulta: 4 febrero 2009].

Toury, G. (2004). Los estudios descriptivos de traducción, y más allá. Metodología de la investigación en estudios de traducción. Traducción, introducción y notas de R. Rabadán y R. Merino, Madrid: Cátedra.

Zaro. J. J. (2007). Shakespeare y sus traductores, Berna: Peter Lang.

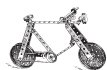

31 